\section{Paralysis of phrenic nerve due to enlargement of left atrium}

Paralysis of the phrenic nerve may be idiopathic or due to bronchogenic carcinoma, tuberculosis, or mediastinal tumours. ${ }^{1}$ We report a case due to enlargement of the left atrium.

\section{Case report}

A 68 year old man was admitted for investigation of a paralysed left hemidiaphragm that had been noted on routine chest radiography. He had a cough productive of a small amount of clear mucoid sputum, moderate exertional dyspnoea, and occasional wheeze but no other respiratory symptoms of note. His medical history included a shrapnel injury sustained in the war, myocardial infarction, and peripheral vascular disease. He had no history of rheumatic fever or tuberculosis. He was receiving oxprenolol, chlorthalidone, potassium chloride, and isoxuprine hydrochloride resinate (a peripheral vasodilator).

Examination showed no finger clubbing, lymphadenopathy, stridor, or obstruction of the superior vena cava. His pulse was 108 beats/minute in sinus rhythm and blood pressure $170 / 90 \mathrm{~mm} \mathrm{Hg}$. Jugular venous pressure was not raised, and heart sounds were soft and difficult to hear because of generalised bilateral expiratory rhonchi. Percussion note was dull at the left base, and inspiration was diminished. The neck and the abdominal and central nervous systems were normal. Chest radiography showed an enlarged left atrium and raised left hemidiaphragm, which was found to be paralysed on sniff testing during fluoroscopy. Bronchoscopy showed no abnormality, and both vocal cords moved symmetrically. Sputum was normal on cytological examination and gave negative results for acid fast bacilli. Erythrocyte sedimentation rate; full blood count; serum concentration of urea, electrolytes, and glucose; and results of liver function tests were normal. Tests for latex-rheumatoid antibody and antinuclear factor were negative, and viral studies showed no rising titre. Electrocardiography showed evidence of $p$ mitrale.

While in hospital he developed a right hemiparesis and aspiration pneumonia and died. Necropsy showed pronounced mitral stenosis (mitral valve circumference $60 \mathrm{~mm})$. The heart was enlarged $(420 \mathrm{~g})$, as were the left ventricle and left atrium (diameter about $80 \mathrm{~mm}$; normal $19-40 \mathrm{~mm}$ ). The lungs showed evidence of aspiration pneumonitis and pulmonary congestion but not of bronchial carcinoma, tuberculosis, mediastinal tumour, or appreciable lymphadenopathy. The left hemidiaphragm was atrophic. The left phrenic nerve was not identifiable in the region adjacent or distal to the left atrium (figure), but the lowest identifiable part was normal on histological examination. The brain showed evidence of cerebral infarction.

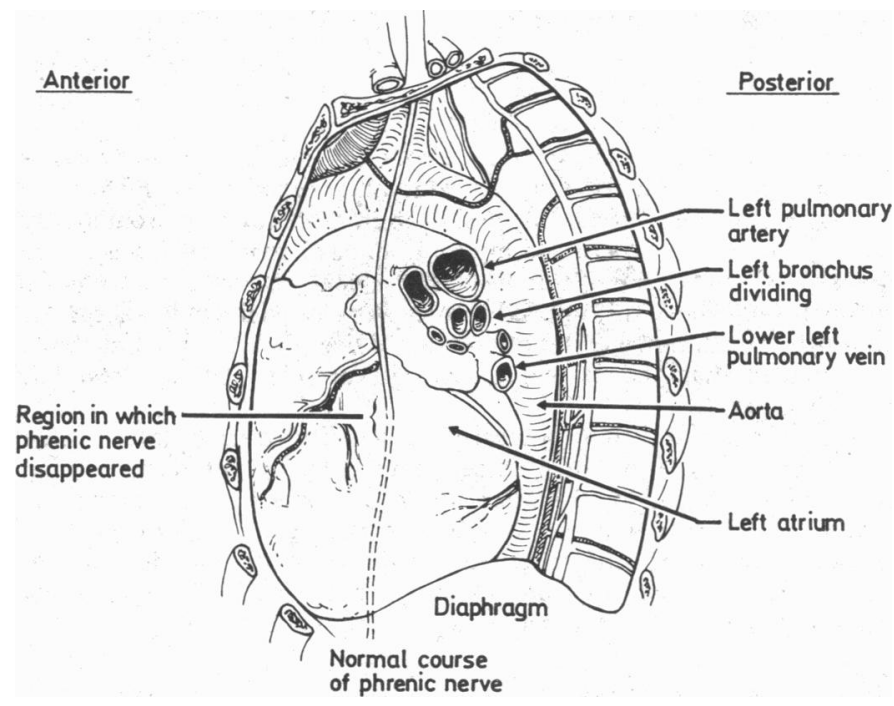

Anteroposterior section of thoracic cavity at necropsy showing enlarged left atrium and normal course of phrenic nerve.

\section{Comment}

Paralysis of the phrenic nerve resulting in a paralysed hemidiaphragm may have various causes including tuberculosis (when it may be iatrogenic after crushing of the phrenic nerve), ${ }^{2}$ bronchial carcinoma, poliomyelitis, herpes zoster, ${ }^{3}$ mediastinitis, diphtheria, tetanus antitoxin, lead poisoning, and trauma; it may also be idiopathic. ${ }^{4}$ Pressure on the phrenic nerve may result from a thoracic aortic aneurysm. ${ }^{5}$ An enlarged left atrium is known to cause recurrent paralysis of the left laryngeal nerve, but we do not know of any report of its causing paralysis of the phrenic nerve, although the anatomical relation of the two structures makes it easy to see how this can happen. As enlarged left atria are common it is surprising that this has not been recorded: possibly some unrecognised factor also contributed in this case. Paralysis of the phrenic nerve due to an enlarged left atrium may be more common than previously thought.

We thank Miss A Scott for typing the manuscript.

' Crofton J, Douglas A. Respiratory diseases. Edinburgh: Blackwell Scientific Publications, 1981.

${ }^{2}$ Gupta SK. Spontaneous paralysis of the phrenic nerve with reference to chronic pulmonary tuberulosis. Br $\mathcal{F}$ Dis Chest 1960;54:283.

${ }^{3}$ Brostoff J. Diaphragmatic paralysis after herpes zoster. $\mathrm{Br}$ Med $\mathcal{F} 1966$; ii:1571.

' Douglas BE, Clagett OT. The prognosis in idiopathic diaphragmatic paralysis. Diseases of the Chest 1960;37:294.

'Sanguinetti AA, Galzerano DA. Sindrome diafragmático por aneurisma de la aorta torácica. Revista de la Asociación Médica Argentina 1943; $57: 413$.

(Accepted 25 fanuary 1984)

Ninewells Hospital and Medical School, Dundee DD1 9SY

W L MORRISON, MRCP, cardiac registrar

$S$ B COGHILL, MB, BMEDSCI, pathologist

King's Cross Hospital, Dundee

R N JOHNSTON, MD, FRCP, consultant chest physician

Correspondence to: Dr W L Morrison.

\section{Interaction between oral contraceptives and griseofulvin}

The Committee on the Safety of Medicines in the United Kingdom and the Netherlands Centre for Monitoring of Adverse Reactions to Drugs have received a total of 22 reports of a possible interaction between oral contraceptives and the antifungal drug griseofulvin.

\section{Analysis of cases}

Twenty women receiving long term oral contraception were reported to have experienced transient intermenstrual bleeding (15 patients; age range 17-42 years) or amenorrhoea (five patients; age range $17-44$ years) in the first or second cycle after beginning treatment with griseofulvin $(0.5-1.0 \mathrm{~g}$ daily). Unintended pregnancies were reported in two other patients.

Seven of the women with intermenstrual bleeding and one with amenorrhoea were using contraceptives containing less than $50 \mu \mathrm{g}$ oestrogen. The patients with amenorrhoea were on average older than those with bleeding $(30.4$ years as against 26.0 years), although the difference was not statistically significant. They were also taking a higher mean daily dose of griseofulvin $(880 \mathrm{mg} v 550 \mathrm{mg}$ ). Fourteen of the 20 women had received no other drugs, three had used drugs that are not known to interfere with oral contraceptives (miconazole ointment, grass pollen vaccine, tetanus vaccine), and in the other three cases details of concomitant drug use were not reported. Four women (two with intermenstrual bleeding, two with amenorrhoea) were re-exposed to griseofulvin and the original reaction recurred.

Of the two women with unintended pregnancies, one had used high dose oral contraception for 15 months and had been taking griseofulvin $500 \mathrm{mg}$ daily for two and a half months. She had also received co-trimoxazole (dose not stated) for one week, beginning one month after starting griseofulvin, and had become pregnant in that period. The second patient had used an unspecified oral contraceptive and was taking griseofulvin and a combination of sulphonamides at the time of conception; no other data were reported.

\section{Comment}

Intermenstrual bleeding, amenorrhoea, and pregnancy may occur spontaneously during the use of oral contraceptives. In our series, however, the temporal relation between beginning griseofulvin and the occurrence of bleeding anomalies, and especially the results of rechallenge with the drug in four patients, are strong evidence of interaction between the oral contraceptives and griseofulvin. 
Griseofulvin modifies hepatic enzyme activity in mice, ${ }^{1}$ so that enzyme induction and a consequent lowering of oestrogen concentrations may explain the intermenstrual bleeding on the basis of a withdrawal effect. Amenorrhoea is not so readily explained. Interestingly, however, rifampicin, a potent inducer of hepatic microsomal enzymes, causes the same range of cycle anomalies with intermenstrual bleeding, amenorrhoea, and unintended pregnancies in women using oral contraceptives. ${ }^{2}$

The contributory role of griseofulvin in the two reported pregnancies is not established, as both women were also taking sulphonamides. These drugs may affect the enzymes concerned in steroid metabolism, at least in vitro, ${ }^{3}$ but are not a well established cause of pill failure.

Pharmacokinetic studies may help in assessing the possible interaction between griseofulvin and oral contraceptives. In the mean time prescribers of these drugs should be alert to the problem, which may lead to pill failure.

We thank Professor Sir Abraham Goldberg for permission to use data from the Adverse Reactions Register of the Committee on the Safety of Medicines.

${ }^{1}$ Denk H, Eckerstorfer R, Talcott RE, Schenkman JB. Alteration of hepatic microsomal enzymes by griseofulvin treatment of mice. Biochem Pharmacol 1977;26:1125-30.

2 Nocke-Finck L, Breuer H, Reimers D. Wirkung von Rifampicin auf den Menstruationszyklus und die Östrogenausscheidung bei Einnahme oraler Kontrazeptiva. Dtsch Med Wochenschr 1973;98:1521-3.

${ }^{3}$ Hempel E, Klinger W. Drug stimulated biotransformation of hormonal steroid contraceptives: clinical implications. Drugs 1976;12:442-8.

(Accepted 12 fanuary 1984)

Netherlands Centre for Monitoring of Adverse Reactions to Drugs, PO Box 439, 2260 AK Leidschendam, the Netherlands

C $P$ H VAN DIJKE, MD, PHD, medical officer

Medicines Division, Department of Health and Social Security, London

J C P WEBER, MD, senior medical officer

Correspondence to: Dr C P H van Dijke.

\section{Fears aroused in patients by migraine}

Pain may arouse fear of death or crippling disease. Migraine may also provoke anxiety about sanity. This was recently brought to my attention by two patients who reluctantly voiced their longstanding dread of mental illness, stimulating me to investigate the nature and prevalence of fears associated with migraine.

\section{Patients and results}

Case 1-A 52 year old housewife had had migraine for 30 years. In describing her attacks she mentioned having hallucinations during the headache phase but hurried on to elaborate on other symptoms. Asked to describe these hallucinations, she said that she had never told anyone about these. With encouragement she explained that she saw objects as being smaller than usual and the wrong way round. When told that Lewis Carroll had had migraine and had probably had similar experiences ${ }^{1}$-hence his creation of Alice-she was obviously relieved, delighted that her visual disturbances were not due to mental derangement.

Case 2-A 44 year old secretary had had classical migraine for 27 years. Her attacks had changed recently, so that she had only the visual aura with no headache. During her headaches she had had difficulty in reasoning and expressing herself. Having been brought up by a schizophrenic uncle, she was terrified that these symptoms heralded schizophrenia.

\section{STUDY}

Fifty one women and 24 men (mean age 46 (range 16-73)) with migraine, as previously defined, ${ }^{2}$ were seen as outpatients at neurological clinics. Some patients mentioned their fears spontaneously during routine questioning $^{3}$; those that did not were asked, "Has your migraine aroused any fear or fears in you ?" Leading questions were purposely avoided. Twenty five patients had no fears. The table lists the fears of the 50 others. Twenty nine patients expressed only one fear each, 12 expressed two fears, eight expressed three, and one expressed four.

Fears aroused by migraine in 50 patients

\begin{tabular}{lc}
\multicolumn{1}{c}{ Fear } & No of patients \\
\hline Work loss (including domestic) & 13 \\
Severity of pain & 8 \\
Frequency or duration of attack & 5 \\
Interference with social activities & 5 \\
Side effects of tablets & 4 \\
Death (severity of attack; progressive weight loss) & 2 \\
Vomiting & 1 \\
Tumour & 14 \\
Stroke & 4 \\
Brain exploding or head bursting & 3 \\
Permanent visual loss & 3 \\
Vision impairing function & 2 \\
Leg paralysis (tingling back of neck) & 1 \\
Insanity & 6 \\
Guilt (failure; helpless) & 3 \\
Mellontophobia & 3 \\
Suicide (because of pain) & 2 \\
Being thought to be mad & 1 \\
Coronary thrombosis (tingling in left hand) & 1 \\
\hline
\end{tabular}

Some patients were unable to specify their fears-for example, four feared damage from taking too many tablets. Others were specific-for example, loss of half a stone in three months due to recurrent attacks with vomiting might progress to death if weight continued to be lost. Fear of death was implied by some patients, consequent on specified fears such as tumour or "head exploding." Three women found that being without a husband accentuated their anxiety. One was a widow, another was divorced, and the third had a husband who was away from home on business.

I suspect that fear of symptoms of mental illness was more common than the patients would admit, many patients being frightened to relate their innermost fears. One patient had returned a present to a friend in the middle of a prolonged migraine when the headache was mild, thus terminating the friendship, and concluded that she must have been insane. Three others feared that they had "something wrong with the brain," implying mental disease. Two patients had contemplated suicide when in severe pain at the height of an attack. A divorcee was frightened because she was solely responsible for her baby. One woman felt "a different person during attacks," which she had had for 39 years. She felt guilty and inadequate because she was unable to cope with her duties as a wife and with looking after her elderly mother; at times she took wrong decisions. Three patients had mellontophobia ${ }^{2}$ - that is, fear of making future arrangements.

\section{Comment}

This study shows that anxieties about organic and mental disease are common in patients with migraine and that these fears may be single or multiple, recent or longstanding, readily or reluctantly voiced, occasional ("it crossed my mind once or twice during a severe attack") or continuous ("terrified of the next attack"), common or unusual, but, above all, unpredictable. Patients visit their doctor for diagnosis, reassurance, explanation, or advice about treatment. This study shows that patients cannot be reassured until their "fear has been ferreted out."

I suspect that fear may increase the severity of migraine. Attacks have been reported in which anxiety caused hyperventilation, adding paraesthesias that made the migraine seem worse. ${ }^{4}$ Furthermore, the expectation of pain may be worse than the actual experience. Removal of fear is an important part of treatment that is derived not from tablets or tests but from an older approach: "The first line of treatment is a word in the patient's ear" (McNeill Love).

1 Klee A. Perceptual disorders in migraine. In: Pearce J, ed. Modern topics in migraine. London: Heinemann Medical, $1975: 45-51$.

2 Blau JN. A plain man's guide to the management of migraine. $\mathrm{Br} \mathrm{Med} \mathrm{F}$ 1982 ;284:1095-7.

${ }^{3}$ Blau JN. How to take a history of head or facial pain. $\mathrm{Br} \mathrm{Med} F \mathrm{f}$ 1982; $285: 1249-51$.

4 Blau JN, Dexter SL. Hyperventilation during migraine attacks. Br Med F $1980 ; 280: 1254$.

(Accepted 19 Fanuary 1984)

National Hospital for Nervous Diseases, London WC1N 3BG

J N BLAU, MD, FRCP, consultant neurologist 